\title{
Trajectory Optimization for Spacecraft Using a Second-Order Gauss Pseudo Spectral Method
}

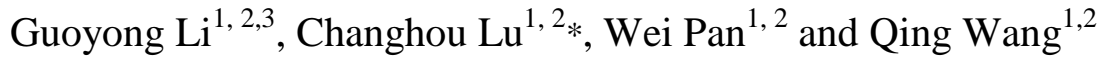 \\ ${ }^{1}$ School of Mechanical Engineering, Shandong University, Jinan, \\ P.R. China, 250061 \\ ${ }^{2}$ Key Laboratory of High-efficiency and Clean Mechanical Manufacture \\ (Shandong University), Ministry of Education, P. R. China \\ ${ }^{3}$ National Network Software Testing Center (Jinan) \\ guoyongli@163.com
}

\begin{abstract}
An optimal control problem for the trajectory of spacecraft under nonlinear constraints is presented. The second-order Gauss pseudo spectral method (GPM) is described for solving the problem numerically. Based on the first-order GPM, the differential matrix of the second-order GPM is derived. The dynamic equations and its constraints are approximated by the two methods. The terminal state constraints of the second-order GPM are converted to two constraints: terminal state variables and the first-order derivative constraints. The firstorder GPM and second-order GPM are compared in terms of the accuracy of the state and control, the number of variables in NLP, the convergence time. A key feature of the secondorder method is that it provides a more accurate and efficient way than the first-order method, due to reducing the number of variables in the nonlinear programming problem that is transcribed. A numerical example is used to identify the key differences between the two methods. The results of this study indicate that the first-order and second-order Gauss methods are very similar in accuracy, while the computational efficiency of the two methods is significantly different, for the numerical solution of nonlinear optimal control problems.
\end{abstract}

Keywords: Nonlinear; Optimal control; LG; Second-order GPM; NLP

\section{Introduction}

Generally for solving optimal control problems in numerical methods, there are two categories: indirect methods and direct methods. In indirect methods, the variational conditions for the optimal problem are typically derived by Hamilton's equation. The optimality conditions are obtained from calculus of variations1 and Pontryagin's minimum principle [1]. Then the optimal problem is transformed into a two-point boundary value problem (TPBVP) solution problem, which is extremely difficult due to the high sensitivity of the Hamiltonian dynamics to unknown boundary conditions. In direct methods, the optimal control problem is directly transcribed to a nonlinear programming problem (NLP) which could be readily solved by a well established optimization method.

In recent years, direct methods for numerically solving complex optimal control problems have become increasingly popular [2]. Pseudospectral methods are a class of direct collocation by discretizing the state variables, control variables and differential-algebraic equations at Gaussian quadrature points. Considerable attention has been focused on

* Changhou Lu: Corresponding author 
pseudospectral methods in the numerical solution of optimal control problems. Pseudospectral methods typically have faster convergence rates and higher accuracy than the others. In the aerospace engineering fields, several well-known pseudospectral methods have been developed such as Gauss pseudospectral method (GPM)[3], Legendre pseudospectral method (LPM) [4], Chebyshev pseudospectral method [5], and Radau pseudospectral method [6].Huntington et al. [7]compared the first three pseudospectral method in terms of the approximation accuracy and computational performance. The results of the study indicate in many applications the performances of the GPM are better than the others. For a high order nonlinear system, the computation is much heavy. If the high order dynamic equations are discretized by the second-order pseudospectral method, the optimization variables and the constraint equations will be less. The computational efficiency may greatly improved by the second-order method. Ross et al. [8] presented a second-order LPM, verified the effectiveness of the method by the numerical simulation. But in the paper the terminal constraints were not given, it is usually difficult to deal with the optimal control problem with terminal constraints by the method. Wen et al. [9] presented a method of costate estimation using a second-order LPM, but the method is base on the first-order LPM, without precise costate estimation theory. But up to now, only a few researches about the second-order pseudo spectral method has deal with, the second-order GPM is more less.

In this paper, we present an optimal control method for the trajectory of spacecraft under complex nonlinear constraints basing on the second-order GMP. The method is used to solve the optimal problem numerically, due to the advantage of accuracy, convergence time, and computational efficiency. The dynamic equations and its constraints are approximated by the first-order GPM and second-order GPM respectively. In a numerical example, the comparison is made between the two methods, in terms of the accuracy of the state and control, the number of variables in NLP, the convergence time.

\section{Optimal Control}

\subsection{Optimal Control Problem}

The paper is devoted to find an optimal trajectory, and the nonlinear optimal control problem is to find the suitable state vectors $x(t)$ and control vectors $u(t)$, satisfying equations (2)(3)(4), while minimizing the following performance indexes.

$$
J=\phi\left(x\left(t_{0}\right), x\left(t_{f}\right)\right)+\frac{1}{2} \int_{t_{0}}^{t_{f}} g(x(t), u(t), t) d t
$$

Subject to the following constraints:

Dynamic equations:

$$
\ddot{x}=f(\dot{x}(t), x(t), u(t), t)
$$

The initial conditions:

$$
\phi(\dot{x}(t=0), x(t=0))=x_{0}
$$

The end conditions:

$$
\phi(\dot{x}(t=f), x(t=f))=x_{f}
$$

Where $x(t)$ are the state variables, $u(t)$ are the control variables, $\dot{x}(t)$ are first-order variables, $\ddot{x}(t)$ are second-order variables. 


\subsection{Second-Order GMP for Optimal Control Problem}

In the paper, the first-order GPM and the second-order GPM are applied for solving the nonlinear optimal control problems; the main fundamental idea is as follows. By a domain transformation technique, the physical horizon is mapped to a computational horizon. Then, the nonlinear optimal control problems are discretized and transcribed to a serial of nonlinear programming problems by the first-order GPM and second-order GPM. The nonlinear programming problems are readily solved by a proper optimization method.

The GPM is based on Legendre-Gauss (LG) points, which lie on the interval $(-1,1)$ and include neither the initial point nor the final point. The optimal control problem presented in the paper is formulated on the interval $\left[\boldsymbol{t}_{\boldsymbol{o}}, \boldsymbol{t}_{f}\right]$, so time domain should be transformed and the affine transformation is as follows.

$$
\tau=\frac{2 t}{t_{f}-t_{0}}-\frac{t_{f}-t_{0}}{t_{f}-t_{0}}
$$

Using the above equation (5), LG points are shifted from the physics domain $\tau \in\left(\tau_{0}, \tau_{f}\right)=($

$-1,1)$. The $N$ discrete points are $(N-2)$ LG points and the initial point, the final point. Both the

Initial point and the end point are assigned; the initial point $\tau_{0}=-1$ corresponds to $t_{0}$, the end

Point $\tau_{f}=+1$ corresponds to $t_{f}$. The physical domain $t \in\left[t_{0}, t_{f}\right]$ completely map to the

Computational domain $\tau \in\left[\tau_{0}, \tau_{f}\right]=[-1,1]$.Equations (1)(2)(3) (4) are expressed respecting

To the computational domain $\tau$.

The minimized performance indexes are transformed into

$$
J=\Phi(x(-1), x(1))+\frac{t_{f}-t_{0}}{2} \int_{-1}^{1} g(x(\tau), u(\tau), \tau) \mathrm{d} \tau
$$

Subject to the conditions:

Second-order dynamic equations:

$$
\ddot{\boldsymbol{x}}(\tau)=\left(\frac{t_{f}-t_{0}}{2}\right)^{2} f\left(\left(\frac{2}{t_{f}-t_{0}}\right) \dot{\boldsymbol{x}}(\tau), \boldsymbol{x}(\tau), \boldsymbol{u}(\tau), \tau\right)
$$

Boundary conditions:

$$
\phi(\dot{x}(-1), \dot{x}(1), x(-1), x(1))=0
$$

Path constraints:

$$
C(\boldsymbol{x}(\tau), \boldsymbol{u}(\tau), \tau) \leq 0
$$

The state and control variables of the optimal control problem are approximated by the Lagrange interpolating polynomials of degree $(N-1)$ at $\mathrm{LG}$ points as

$$
\begin{aligned}
& x\left(\tau_{k}\right) \approx X\left(\tau_{k}\right)=\sum_{i=0}^{N-2} L_{i}\left(\tau_{k}\right) X\left(\tau_{i}\right) \\
& u\left(\tau_{k}\right) \approx U\left(\tau_{k}\right)=\sum_{i=0}^{N-2} L_{i}\left(\tau_{k}\right) U\left(\tau_{i}\right)
\end{aligned}
$$

Where $\boldsymbol{X}\left(\tau_{k}\right)$ is the state approximation at $\tau_{k}$ point $U\left(\tau_{k}\right)$ is the control approximation at $\tau_{k}$ point. $\tau_{k}(\mathrm{k}=1 \ldots N-2)$ is the LG points, $\tau_{i}(\mathrm{k}=0 \ldots N-2)$ are $\tau_{k}$ plus the initial point $\tau_{0}=-1$. 
$L_{i}(\tau)$ Is the basic functions of (N-1) Lagrange interpolation polynomials on the interval [-1, 1]. The LG points are the roots of the Nth degree Legendre polynomials, $P_{N}(\tau)$.

$$
L_{i}(\tau)=\prod_{\substack{j=0 \\ i \neq j}}^{N-2} \frac{\tau-\tau_{j}}{\tau_{i}-\tau_{j}}, \quad i=0 \ldots N-2
$$

The first-order derivate and second-order derivate constraints are transformed into algebraic equations using the differential matrix, as below.

$$
\begin{aligned}
& \dot{\boldsymbol{x}}\left(\tau_{k}\right) \approx \dot{\boldsymbol{X}}\left(\tau_{k}\right)=\sum_{i=0}^{K} \dot{L}_{i}\left(\tau_{k}\right) \boldsymbol{X}\left(\tau_{i}\right)=\sum_{i=0}^{K} D_{k i} \boldsymbol{X}\left(\tau_{i}\right) \\
& \ddot{\boldsymbol{x}}\left(\tau_{k}\right) \approx \ddot{\boldsymbol{X}}\left(\tau_{k}\right)=\sum_{i=0}^{K} \ddot{L}_{i}\left(\tau_{k}\right) \boldsymbol{X}\left(\tau_{i}\right)=\sum_{i=0}^{K} S_{k i} \boldsymbol{X}\left(\tau_{i}\right)
\end{aligned}
$$

Where $\boldsymbol{D}_{k, i}$ are entries of the $N \times(N+1)$ first-order differential matrix, $D_{k i}=\dot{L}_{i}\left(\tau_{k}\right)$ [10]; $S_{k, i}$ are entries of the $N \times(N+1)$ second-order differential matrix, $S_{k i}=\ddot{L}_{i}\left(\tau_{k}\right)$. They have one row for LG points, the elements in the $i$ th column are the first-order or second-order derivatives of the Lagrange polynomials evaluated at each of the collocation points.

$$
\begin{gathered}
D_{k i}= \begin{cases}\frac{\left(1+\tau_{k}\right) \dot{P}_{K}\left(\tau_{k}\right)+P_{K}\left(\tau_{k}\right)}{\left(\tau_{k}-\tau_{i}\right)\left[\left(1+\tau_{i}\right) \dot{P}_{K}\left(\tau_{i}\right)+P_{K}\left(\tau_{i}\right)\right]}, & (i \neq k) \\
\frac{\left(1+\tau_{i}\right) \ddot{P}_{K}\left(\tau_{i}\right)+2 \dot{P}_{K}\left(\tau_{i}\right)}{2\left[\left(1+\tau_{i}\right) \dot{P}_{K}\left(\tau_{i}\right)+P_{K}\left(\tau_{i}\right)\right]}, & (i=k)\end{cases} \\
S_{k i}= \begin{cases}\frac{\left(1+\tau_{k}\right)\left(\tau_{k}-\tau_{i}\right) \ddot{P}_{K}\left(\tau_{k}\right)-2\left(1+\tau_{i}\right) \dot{P}_{K}\left(\tau_{k}\right)}{\left(\tau_{k}-\tau_{i}\right)^{2}\left[\left(1+\tau_{i}\right) \dot{P}_{K}\left(\tau_{i}\right)+P_{K}\left(\tau_{i}\right)\right]}, & (i \neq k) \\
\frac{\left(1+\tau_{i}\right) \dddot{P}_{K}\left(\tau_{i}\right)+3 \ddot{P}_{K}\left(\tau_{i}\right)}{3\left[\left(1+\tau_{i}\right) \dot{P}_{K}\left(\tau_{i}\right)+P_{K}\left(\tau_{i}\right)\right]}, & (i=k)\end{cases}
\end{gathered}
$$

$\tau_{k}(K=1, N-2)$ are the LG points, neither $\tau_{0}$ or $\tau_{N-1} \cdot \tau_{i}(K=0 \ldots N-2)$ are LG points and the initial point, $P_{K}$ is $k t h$ degree Legendre polynomials.

$$
\sum_{i=0}^{K} S_{k i} \boldsymbol{X}\left(\tau_{i}\right)-\left(\frac{t_{f}-t_{0}}{2}\right)^{2} \boldsymbol{f}\left(\left(\frac{2}{t_{f}-t_{0}}\right) \sum_{i=0}^{K} D_{k i} \boldsymbol{X}\left(\tau_{k}\right), \boldsymbol{X}\left(\tau_{k}\right), \boldsymbol{U}\left(\tau_{k}\right), \tau_{k}\right)=0
$$

An integral of a function is approximated by the Gauss quadrature, and then the integral constraints are transformed into a set of algebraic equations. The general form for the quadrature approximation in the GPM is

$$
\int_{a}^{b} f(\tau) d \tau \approx \sum_{i=0}^{N} w_{i} f\left(\tau_{i}\right)
$$

Where $w_{i}$ is the Gauss quadrature integration weights given by

$$
w_{i}=\frac{1}{\left(1-\tau_{i}^{2}\right)} \cdot \frac{2}{\left[\dot{P}_{N}\left(\tau_{i}\right)\right]^{2}}
$$


By the Gauss-Lobatto integral rule which provides a highly accurate result, the integral in the performance index is approximated and equations (6) can be expressed by the following form:

$$
J=\Phi\left(\boldsymbol{X}\left(\tau_{f}\right), \tau_{f}\right)+\frac{t_{f}-t_{0}}{2} \sum_{k=1}^{N-1} w_{k} g\left(\boldsymbol{X}\left(\tau_{k}\right), \boldsymbol{U}\left(\tau_{k}\right), \tau_{k}\right)
$$

Constraint equations (7) can be expressed:

$$
\dot{\boldsymbol{X}}\left(\tau_{f}\right)-\dot{\boldsymbol{X}}\left(\tau_{0}\right)-\frac{t_{f}-t_{0}}{2} \sum_{k=1}^{N-1} w_{k} \boldsymbol{f}\left(\left(\frac{2}{t_{f}-t_{0}}\right) \sum_{i=0}^{N-1} D_{k i} \boldsymbol{X}\left(\tau_{k}\right), \boldsymbol{X}\left(\tau_{k}\right), \boldsymbol{U}\left(\tau_{k}\right), \tau_{k}\right)=0
$$

Where $\boldsymbol{X}\left(\tau_{0}\right)$ and $\boldsymbol{X}\left(\tau_{f}\right)$ is the initial constraint and the terminal constraint, $\dot{\boldsymbol{X}}\left(\tau_{0}\right)$ and $\dot{\boldsymbol{X}}\left(\tau_{f}\right)$ is the first-order derivate. From equation (21), the first-order derivate of a discrete point $\dot{\boldsymbol{X}}\left(\tau_{k}\right)$ is expressed by the sum $\dot{\boldsymbol{X}}\left(\tau_{0}\right)$ add $k-l$ Gauss integral equations, as below :

$$
\dot{\boldsymbol{X}}\left(\tau_{k}\right)=\dot{\boldsymbol{X}}\left(\tau_{0}\right)+\frac{t_{f}-t_{0}}{2} \sum_{j=1}^{k-1} \omega_{k} \boldsymbol{f}\left(\left(\frac{2}{t_{f}-t_{0}}\right) \sum_{i=0}^{N-2} D_{j i} \boldsymbol{X}\left(\tau_{j}\right), \boldsymbol{X}\left(\tau_{j}\right), \boldsymbol{U}\left(\tau_{j}\right), \tau_{j}\right)
$$

When $k=1, \quad \dot{\boldsymbol{X}}\left(\tau_{1}\right) \approx \dot{\boldsymbol{X}}\left(\tau_{0}\right)$

From equation (22) (23), the terminal constraints of the second-order GPM is expressed as

$$
\boldsymbol{X}\left(\tau_{f}\right)-\boldsymbol{X}\left(\tau_{0}\right)-\frac{t_{f}-t_{0}}{2} \sum_{k=1}^{K} \omega_{k} \dot{\boldsymbol{X}}\left(\tau_{k}\right)=0
$$

The initial constraint is expressed as:

$$
\boldsymbol{X}\left(\tau_{0}\right)=\boldsymbol{X}_{0}
$$

The path constraint is expressed as:

$$
\boldsymbol{h}\left(\boldsymbol{X}\left(\tau_{k}\right), \boldsymbol{U}\left(\tau_{k}\right), \tau_{k}\right) \leq 0
$$

The nonlinear trajectory optimal problem that is described by equations (1) (2) (3) (4) has been discretized and transformed into a NLP by the GPM. Many NLP solvers are available for solving the problem routinely, such as Sequential quadratic programming, variable metric method. The optimal control law is obtained from the solution.

\section{Numerical Example}

The application of GPM in spacecraft orbital transfer with the optimal time problem is studied; the performance of second-order GPM is verified. These problem dynamic equations are modeled by the second Lagrange equation in polar coordinates. The optimal control problem is described as follows, minimizing the cost function.

$$
J=\int_{t_{0}}^{t_{f}} \mathrm{~d} t=t_{f}-t_{0}
$$

The second-order dynamic equations constraints

$$
\left\{\begin{array}{l}
\ddot{r}=\dot{\theta}^{2} r-\frac{\mu}{r^{2}}+\frac{a T_{r}}{m_{0}-\dot{m} t} \\
\ddot{\theta}=-\frac{2 \dot{\theta} \dot{r}}{r}+\frac{a T_{\theta}}{\left(m_{0}-\dot{m} t\right) r}
\end{array}\right.
$$


Where $m_{0}$ is the initial mass of the space vehicle, $\dot{m}$ is the constant fuel consumption rate, $r$ is the orbit radius, $\theta$ is the orbital angle, $\mu$ is the gravitational parameter, $T$ is the thrust magnitude, $a$ is the thrust. The parameters are given as follows in normalized form.

$$
m_{0}=1, \quad \dot{m}=0.0749, \quad a=0.1405
$$

The boundary conditions

$$
\left\{\begin{array} { l } 
{ r _ { 0 } = 1 } \\
{ \theta _ { 0 } = 0 } \\
{ \dot { r } _ { 0 } = 0 } \\
{ \dot { \theta } _ { 0 } = 1 }
\end{array} \quad \left\{\begin{array}{l}
r_{f}=1.524 \\
\theta_{f}=\text { free } \\
\dot{r}_{f}=0 \\
\dot{\theta}_{f}=0.5315
\end{array}\right.\right.
$$

When the problem is appropriated by second-order GPM, the dimensionless state vector is $x=(r, \theta)$. While the problem is appropriated by first-order GPM, the second order.

Derivate dynamic equations should be translated to first-order derivate form as blow.

$$
\left\{\begin{array}{l}
\dot{r}=u \\
\dot{\theta}=\frac{v}{r} \\
\dot{u}=\frac{v^{2}}{r}-\frac{\mu}{r^{2}}+\frac{a T_{r}}{m_{0}-\dot{m} t} \\
\dot{v}=-\frac{u v}{r}+\frac{a T_{\theta}}{m_{0}-\dot{m} t}
\end{array}\right.
$$

Where $u$ is the radial velocity, $v$ is the tangential velocity, in the first-order derivate form the dimensionless state vector is $x=(r, \theta, u, v)$. It is obvious that the number of variables of second-order GMP is less than first-order, then the scale of the NLP that is directed by second-order GMP smaller, the computational efficiency higher.

A comparison is made between the first-order GPM and the second-order GPM to solve the optimal control in the example. In the studies, the initial conditions and the end conditions are the same in two methods. It is clear that increasing the number of the nodes can improve the actuary, but it comes at a significant computational burden. Proper the number of nodes should be chosen, $50 \mathrm{LG}$ points are used to discretize the problem. In computing, once the error drops below the tolerances of the NLP, the convergence rate will stop improving. In simulate software, the error tolerances of the function iteration are set to 1e-7. The simulation results are as below.

Figure 1 shows the time history of the optimal state variables $r, \theta$, under the first-order GPM and the second-order GPM. The left is the whole graph; the right is the detailed drawing. 

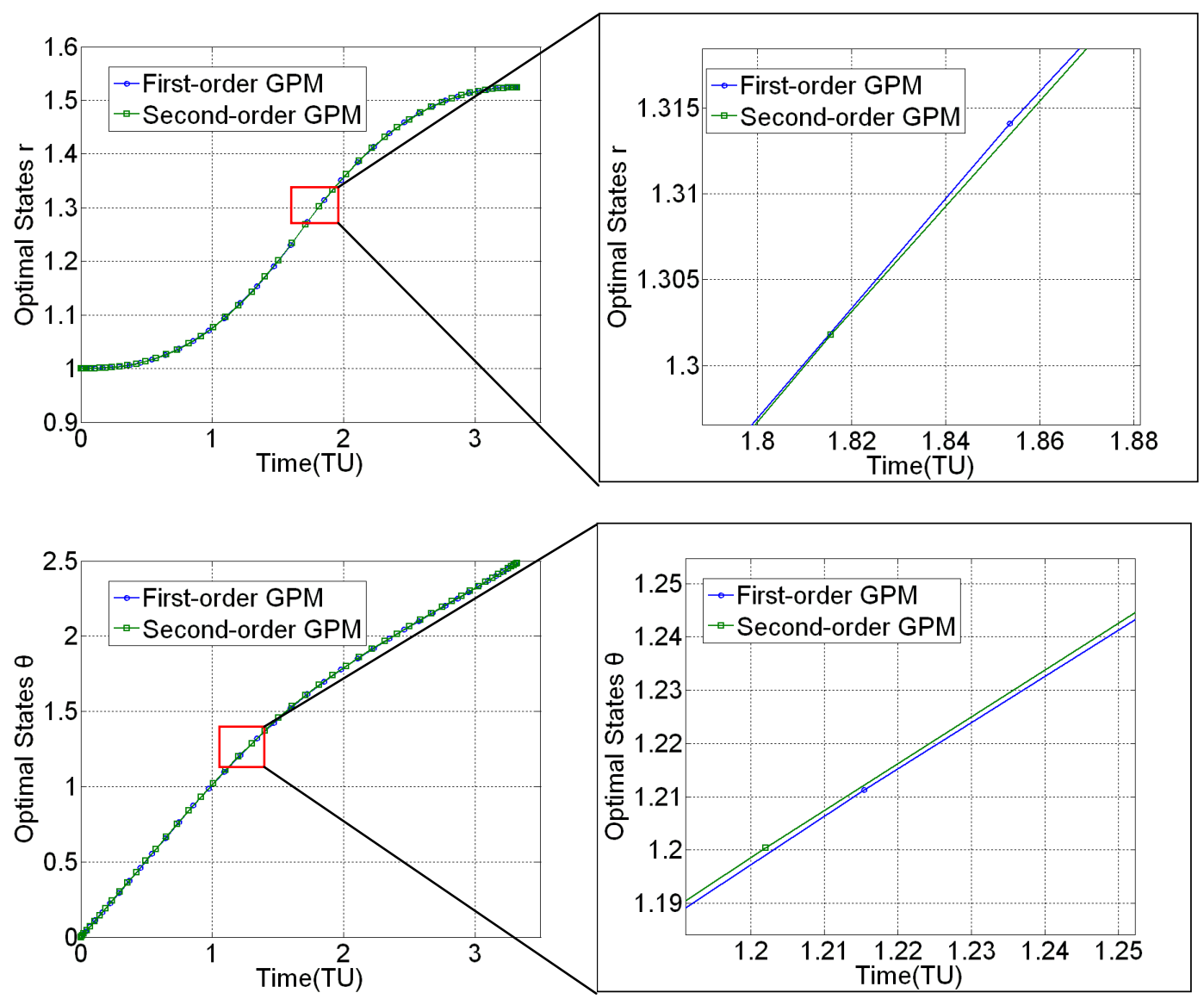

Figure 1. Optimal States r, $\theta$ VS Time

Figure 2 shows the time history of the optimal control input $\operatorname{Tr}, T_{\theta}$ under the first-order GPM and the second-order GPM.
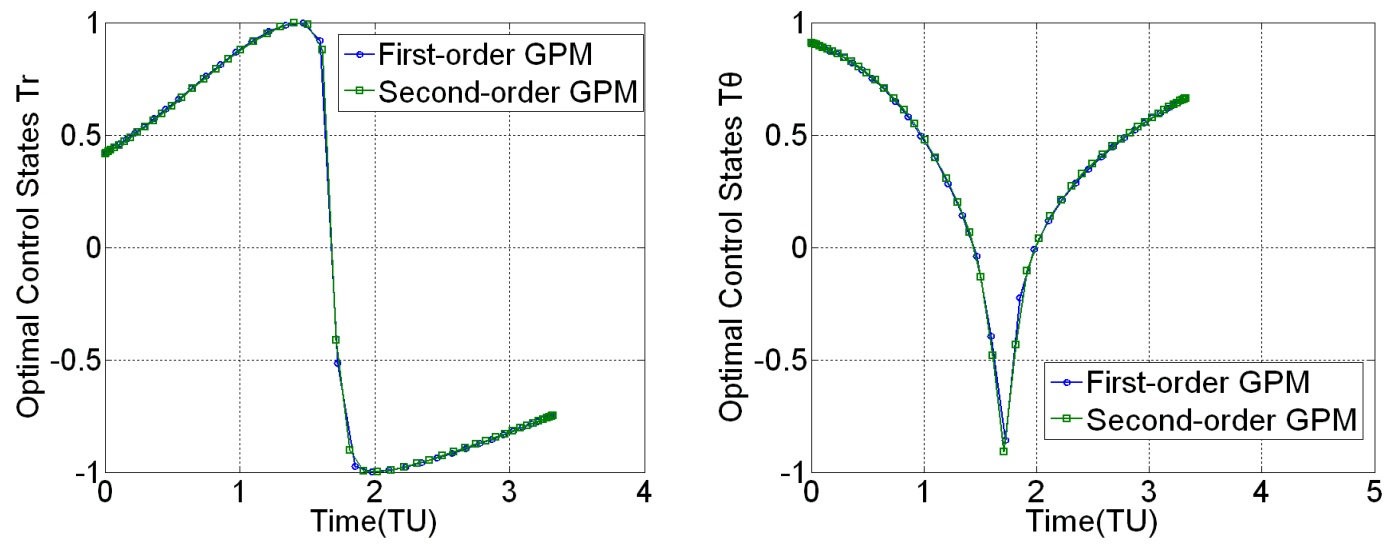

Figure 2. Optimal Control Input TR, $T_{\theta}$ VS Time 
Figure 3 shows the change of the number of variables in NLP with the nodes increasing, under the first-order GPM and the second-order GPM.

Figure 4 shows the convergence time with the nodes increasing, under the first-order GPM and the second-order GPM.

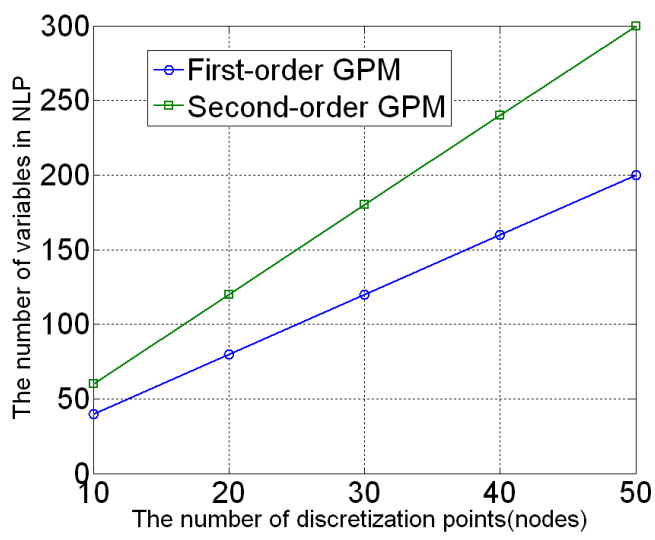

Figure 3. The Number of Variables VS Nodes

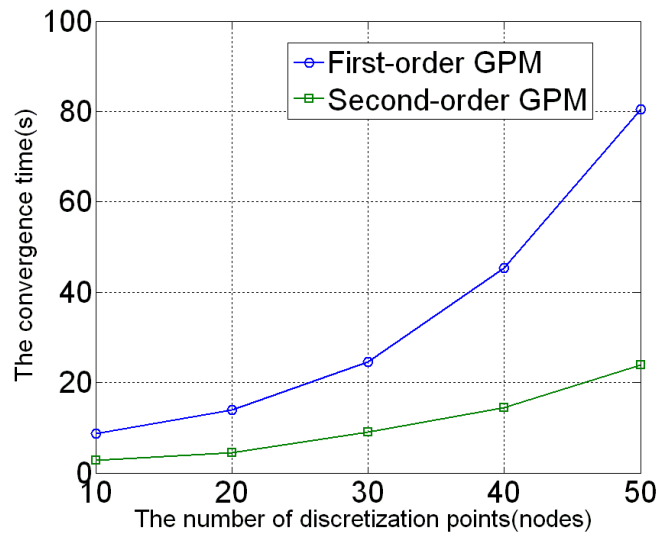

Figure 4. The Convergence Time VS Nodes

Figure 1-2 shows the state and control variables between first-order GPM and secondorder GPM, have a slight difference, and the changing trend is almost the same. The Optimize trajectories from the two methods are almost the same, then the validity of the second-order GPM is verified. Fig 3 shows the change of the number of variable in NLP with the nodes increasing. With the increase of discrete points, the first-order GPM of variable number increases relatively quickly, the scale of nonlinear programming become larger. Fig 4 shows the change of convergence time with the nodes increasing. It is obvious that when the number of nodes is small, the computing time between the two methods has a slight difference. With the nodes increasing, the difference becomes considerably distinct. When the number of nodes in the problem is increased to 50, the second-order GPM has a great advantage. The computing time of the second-order GPM is greatly less than that of the first-order GPM, Only one fourth.

\section{Conclusions}

The method using second-order GPM to solve the numerical solution of nonlinear optimal control problems is presented. The first-order GPM and second-order GPM have been compared in terms of the accuracy of the state and control, the number of variables in NLP, the convergence time. In the study, an example is used to identify the key differences between the two methods. The results of the study indicate that the accuracy of the first-order GPM and second-order GPM methods are very similar, but the computational efficiency of the two methods is significantly different. With the nodes increasing, the advantage of the secondorder GPM is more obvious. The second-order GPM retains the accuracy as the first-order while greatly reduces the computation time and improves the computational efficiency due to reducing the number of variables and constraint equations in NLP. 


\section{Acknowledgements}

This work is supported by the National Equipment Pre-research Fund of China under Grant 62501040402 .

\section{References}

[1] A. E. Bryson, "Dynamic Optimization", Addison-Wesley, Menlo Park, CA, (1999), pp. 315-318.

[2] J. T. Betts, "Survey of Numerical Methods for Trajectory Optimization", Journal of Guidance, Control, and Dynamics, vol. 21, no. 2, (1998), pp. 193-207.

[3] D. A. Benson, G. T. Huntington, T. P. Thorvaldsen, and A. V. Rao, "Direct Trajectory Optimization and Costate Estimation via an Orthogonal Collocation Method", Journal of Guidance, Control, and Dynamics, vol. 29, no. 6, (2006), pp. 1435-1440.

[4] F. Fahroo and I. M. Ross, "Co-state estimation by a Legendre pseudospectral method", Journal of Guidance, Control, and Dynamics, vol. 24, no. 2, (2001), pp. 270-277.

[5] Garg, P. Divya, A. Michael, C. Francolin, "Direct trajectory optimization and costate estimation of finitehorizon and infinite-horizon optimal control problems using a Radau pseudospectral method", computational optimization and applications, vol. 49, no. 2, (2011), pp. 335-358.

[6] G. T. Huntington, D. Benson and A. V. Rao, "A Comparison of Accuracy and Computational Efficiency of three Pseudospectral Methods", AIAA Guidance, Navigation and Control Conference and Exhibit, South Carolina, (2007).

[7] I. M. Ross and F. Fahroo, "Exploiting higher-order derivatives in computational optimal control", The 10th Mediterranean Conference on Control and Automation, Lisbon, Portugal, (2002).

[8] H. Wen, D. P. Jin and H. Y. Hu, "Costate estimation for dynamic systems of the second order", Science in China Series E: Technological Sciences, vol. 52, no. 3, (2009), pp. 752-760.

[9] G. Karniadakis and S. Sherwin, "Spectral/hp Element Methods for Computational Fluid Dynamics", Oxford Univ. Press, Oxford (2005).

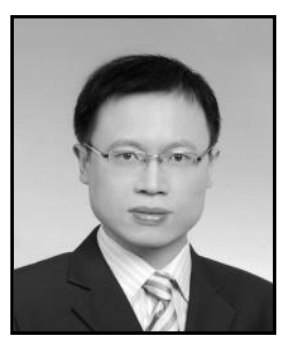

Guoyong Li, $\mathrm{He}$ is currently pursuing the $\mathrm{Ph} . \mathrm{D}$. degree of mechatronic engineering at School of Mechanical Engineering, Shandong University. He received the M.S. degree from the school of automation, Beijing University of posts and telecommunications. His research interests includes nonlinear control, optimal control of spacecraft. He has also been worked in National Network Software Testing Center (Jinan).

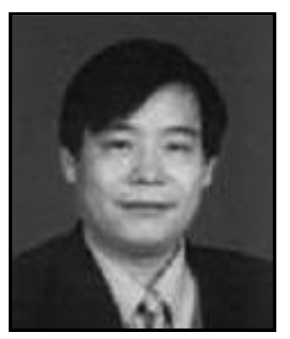

Changhou Lu, He is currently a professor and a doctoral supervisor of mechatronic engineering at School of Mechanical Engineering of Shandong University. He has published over 120 papers on vibration control, trace control and high-speed mechanical control. He has successfully completed over 20 research projects supported by national and ministry funds. 


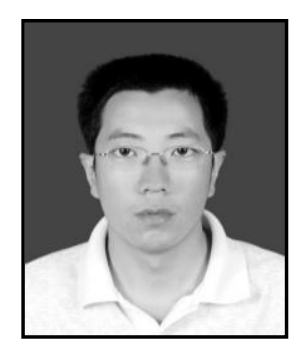

Wei Pan, He is currently a Lecturer of mechatronic engineering at School of Mechanical Engineering, Shandong University. He received the M.S. and Ph.D. degrees from Shandong University, both in mechatronic engineering, in 2001 and 2010, respectively. His research interest includes optimal control, low-thrust orbital maneuvers, cooperative control of autonomous vehicles, space maneuvers for tethered systems and industry applications of control theory.

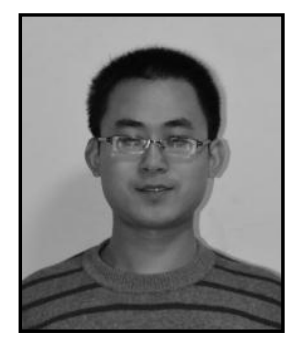

Qing Wang, He is currently pursuing the Ph.D. degree of mechatronic engineering at School of Mechanical Engineering, Shandong University. He received the B.E. degree in manufacturing science and engineering from Sichuan University, Sichuan,China, in 2011. His research interests included nonlinear control system, adaptive control, and distributed parameter control system. 